\title{
Mucocele oral provocada por mordida acidental: relato de caso
}

Oral mucocele caused by accidental bite: case report

\author{
Mucocele bucal causada por la mordedura accidental: reporte de caso
} Bruno Firmino de OLIVEIRA

Douglas Benício Barros HENRIQUE José Henrique de Araújo $\mathbf{C R U Z}^{2}$

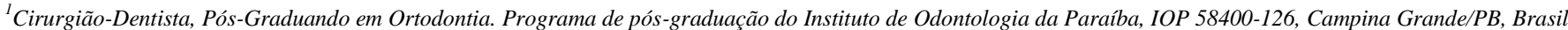
${ }^{2}$ Acadêmico do Curso de Graduação em Odontologia. Centro de Saúde e Tecnologia Rural, Universidade Federal de Campina Grande, UFCG 58708-110 Patos/PB, Brasil

\section{Resumo}

Mucocele é definida como fenômeno de retenção ou extravasamento de muco. A mucocele oral é provocada por traumatismos mecânicos, que comprimem e vedam o ducto das glândulas salivares menores, impossibilitando a secreção de saliva, mesmo sua produção continuando a ocorrer, fazendo com que haja aumento de volume da glândula e ducto envolvidos. Existem várias modalidades de tratamento que incluem excisão cirúrgica da lesão, laserterapia, criocirurgia, escleroterapia, micromarsupialização, injeção intralesional de corticosteroide ou agente esclerosante, além da regressão espontânea. A remoção cirúrgica convencional das mucoceles utilizando um bisturi é considerada a opção mais comum e requer a ressecção completa da lesão e glândulas salivares menores associadas para diminuir o risco de recidiva. O presente trabalho relata um caso de excisão cirúrgica de mucocele em mucosa labial inferior causada por mordida acidental, realizada em paciente atendido na clínica de Odontologia do Centro de Saúde Odontominas, Patos/PB. Diante disso, discute sobre o diagnóstico e as diferentes terapias (cirúrgicas e não cirúrgicas) para o tratamento de mucocele. Após a remoção cirúrgica da lesão, os resultados se mostraram satisfatórios, com pós-operatório indolor, sem edema ou quaisquer outras queixas por parte do paciente. Não houveram recidivas ou aparecimento de novas lesões. A remoção completa da mucocele e glândulas salivares acessórias, bem como a ausência de recidivas, caracterizou o sucesso na abordagem do caso.

Descritores: Mucocele; Cirurgia Bucal; Diagnóstico Bucal.

\section{Abstract}

Mucocele is defined as a phenomenon of retention or extravasation of mucus. The oral mucocele is caused by mechanical trauma, which compress and seal the duct of the minor salivary glands, preventing the secretion of saliva, even their production while continuing to occur, so that there is an increase of volume of the gland and ductus involved. There are several treatment modalities which include surgical excision of the lesion, laser therapy, cryosurgery, sclerotherapy, micromarsupialização, intralesional injection of corticosteroids or sclerosing agent, besides spontaneous regression. The conventional surgical removal of mucoceles using a scalpel is considered the most common option and requires a complete resection of the lesion and minor salivary glands associated to decrease the risk of recurrence. The present study reports a case of surgical excision of salivary mucocele in lower labial mucosa caused by accidental bite, held in patient in the clinic of Dentistry of the Centro de Saúde Odontominas, Patos/PB. In addition, discusses the diagnosis and the different therapies (surgical and non surgical procedures) for the treatment of mucoceles. After the surgical removal of the lesion, the results were satisfactory, with post-operative pain, without edema or any other complaints on the part of the patient. There were no recurrences or appearance of new lesions. The complete removal of the mucocele and ancillary salivary glands, as well as the absence of relapse characterized the success in the approach of the case.

Descriptors: Mucocele; Surgery, Oral; Diagnosis, Oral.

\section{Resumen}

Mucocele es definido como un fenómeno de retención o extravasación de moco. El mucocele oral es causado por el trauma mecánico, que comprimir y sellar el conducto de las glándulas salivales menores, evitando la secreción de saliva, incluso su producción mientras continúa produciéndose, por lo que hay un aumento de volumen de la glándula y el ductus involucrados. Existen varias modalidades de tratamiento que incluyen la escisión quirúrgica de la lesión, la terapia con láser, la criocirugía, la escleroterapia micromarsupialização, inyección intralesional de corticoides o agente esclerosante, además de la regresión espontánea. La extirpación quirúrgica convencional de los mucoceles utilizando un escalpelo es considerada la opción más común y requiere una resección completa de la lesión y de glándulas salivales menores asociados a disminuir el riesgo de recurrencia. El presente estudio se reporta un caso de escisión quirúrgica del mucocele salival en la mucosa labial inferior causada por la picadura accidental, celebrada en pacientes en la clínica de Odontología del Centro de Saúde Odontominas, Patos/PB. Además, discute el diagnóstico y las diferentes terapias (procedimientos quirúrgicos y no quirúrgicos) para el tratamiento de los mucoceles. Después de la extirpación quirúrgica de la lesión, los resultados fueron satisfactorios, con dolor postoperatorio, sin edema o cualquier otras quejas por parte de la paciente. No hubo recidivas o la aparición de nuevas lesiones. La extracción completa del mucocele y glándulas salivares accesorias, así como la ausencia de recidiva caracteriza el éxito en el método del caso.

Descriptores: Mucocele; Cirugía Bucal; Diagnóstico Bucal.

\section{INTRODUÇÃO}

Mucocele é definida como fenômeno de retenção ou extravasamento de muco que pode aparecer na cavidade oral, apêndice, vesícula biliar, seios paranasais, ou saco lacrimal. As lesões orais são resultantes do acúmulo de saliva, com ou sem extravasamento, devido a alterações nas glândulas salivares menores ${ }^{1,2}$. Representam uma das formas mais comuns de lesões de mucosa oral, acometendo locais da onde o epitélio é menos ceratinizado como língua, mucosa jugal, assoalho oral (sendo denominada de rânula) e, com maior frequência, a mucosa labial inferior ${ }^{3,4}$.
A mucocele oral é provocada por traumatismos mecânicos, que comprimem e vedam o ducto das glândulas salivares menores, impossibilitando a secreção de saliva, mesmo sua produção continuando a ocorrer, fazendo com que haja aumento de volume da glândula e ducto envolvidos ${ }^{5}$. Os fatores mais comumente associados à formação de mucocele oral são a mordida acidental da mucosa ou algum hábito parafuncional. Outras razões são os fatores irritantes, como, por exemplo, lesões causadas por aparatos de aparelho ortodôntico ou prótese, giroversões dentárias e diastemas ${ }^{2,6,7}$. 
É clinicamente caracterizada por uma lesão única de coloração semelhante à mucosa circundante ou levemente azulada, indolor, suave e lisa à palpação, com formato esférico, podendo apresentarse como nódulo flutuante ${ }^{8}$. O diâmetro das mucoceles varia de poucos milímetros até centímetros ${ }^{9}$ e sua incidência é difícil de ser estimada devido ao fato de que uma grande percentagem dessas lesões não é encaminhada para exame histopatológico ${ }^{10}$.

Geralmente, são classificadas em dois tipos: de retenção e de extravasamento. Mucocele de extravasamento é resultante de um ducto de glândula salivar traumatizado, com consequente derramamento de saliva nos tecidos moles ao redor da glândula, que pode também sofrer retenção de muco salivar devido ao bloqueio dos ductos da glândula salivar menor associada. Esse tipo de lesão está mais presente no lábio inferior. Já a mucocele de retenção geralmente está localizada na bochecha ou palato de pacientes mais velhos, completamente revestida por epitélio e sem sofrer o fenômeno de extravasamento de muco salivar, ocorrendo com menos frequência ${ }^{11,12}$.

Existem várias modalidades de tratamento que incluem excisão cirúrgica da lesão, laserterapia, criocirurgia, escleroterapia, micromarsupialização, injeção intralesional de corticosteróide ou agente esclerosante $^{8}$, além da regressão espontânea. A remoção cirúrgica convencional das mucoceles utilizando um bisturi é considerada a opção mais comum e requer a ressecção completa da lesão e glândulas salivares menores associadas para diminuir o risco de recidiva ${ }^{2}$.

O presente trabalho relata um caso de excisão cirúrgica de mucocele em mucosa labial inferior causada por mordida acidental realizada em paciente atendido na clínica de Odontologia do Centro de Saúde Odontominas, Patos/PB. Diante disso, discute sobre o diagnóstico e as diferentes terapias (cirúrgicas e não cirúrgicas) para o tratamento de mucocele.

\section{CASO CLÍNICO}

Paciente E.P.C., 37 anos de idade, gênero masculino, feoderma, procurou atendimento odontológico no Centro de Saúde Odontominas (Patos-PB), referindo "bolha no lábio que aumentava e diminuía ao longo do dia". Durante a anamnese, foi relatado o surgimento da lesão após mordedura acidental executada durante uma refeição e que persistia há cerca de dois meses. Por esse período, a lesão apresentou-se assintomática, sem nenhuma alteração sistêmica decorrente, com volume variante ao longo do dia. Descreveu-se também que a lesão nunca regredira por completo, mas que sempre durante $\mathrm{o}$ aumento de volume ocorria $\mathrm{o}$ extravasamento de líquido após ser rompida pelo próprio paciente.

Ao exame clínico intraoral foi constatado lesão com cerca de $4 \mathrm{~mm}$ de diâmetro, na região mediana da mucosa labial inferior, de aspecto bolhoso, bem delimitada e de base séssil, superfície lisa e flutuante à palpação, com coloração semelhante à mucosa circunjacente (Figura 1). A hipótese diagnóstica foi de mucocele e a conduta terapêutica compreendeu a remoção cirúrgica da lesão (biópsia excisional) e glândulas associadas.

Após antissepsia extra-oral com clorexidina a $2 \%$ (Riohex, Rioquímica ${ }^{\circledR}$, Brasil) intra-oral através do bochecho com solução de clorexidina a $0,2 \%$ (Periogard, Colgate ${ }^{\circledR}$, Brasil), o procedimento cirúrgico foi realizado com anestesia local infiltrativa (Figura 2) com Cloridrato de Lidocaína a 2\% associado a Epinefrina 1:100.000 (Alphacaine, Nova DFL $^{\circledR}$, Brasil).

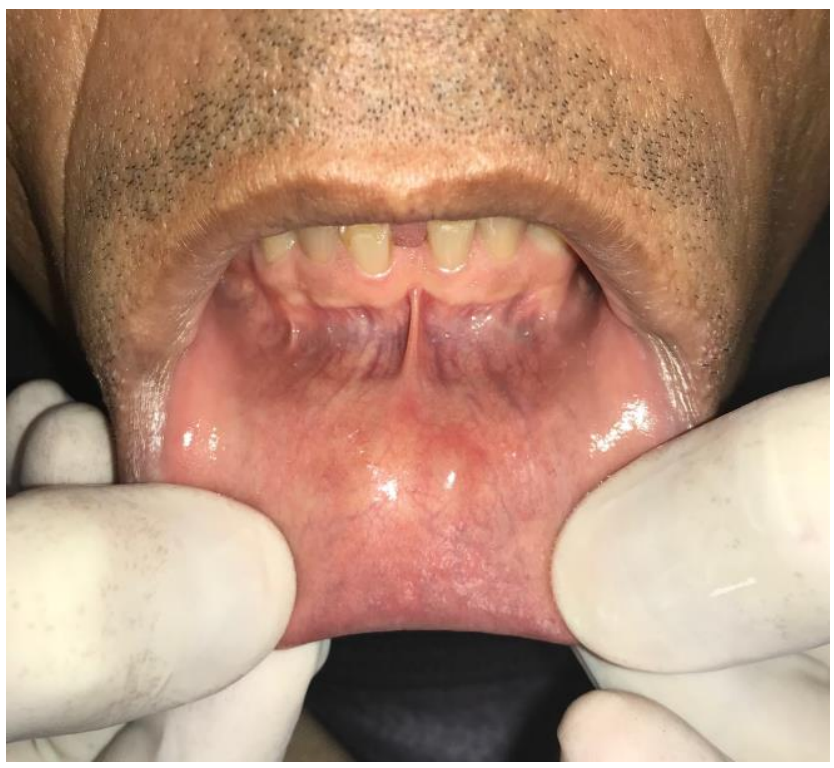

Figura 1: Aspecto clínico da mucocele na mucosa labial inferior.

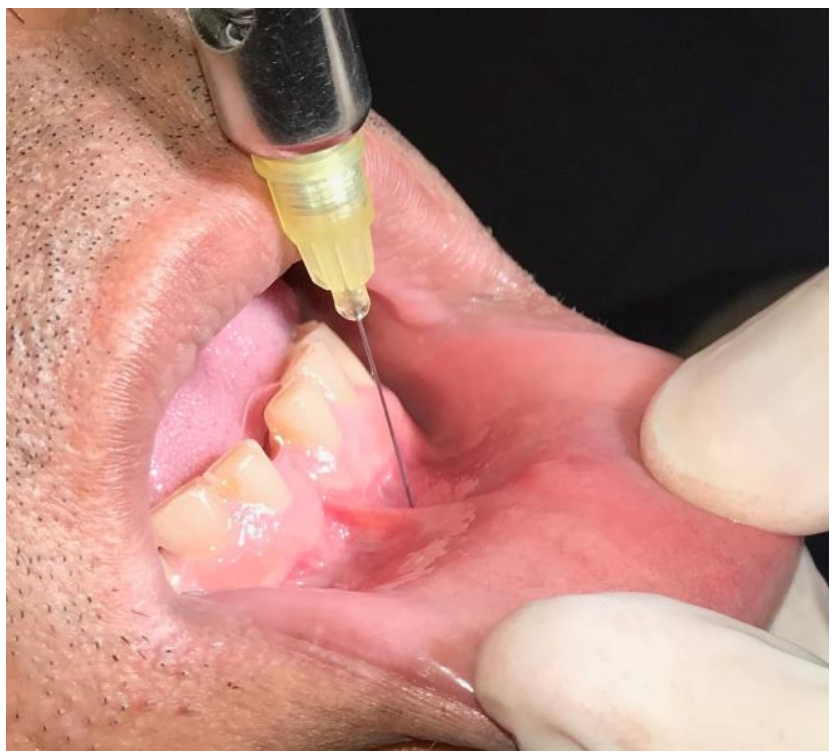

Figura 2: Técnica anestésica local.

Seguiu-se a incisão em forma de elipse com margem de segurança (Figuras 3 e 4) utilizando lâmina de bisturi de aço inox estéril $\mathrm{N}^{\circ} 15$ (Lamedid 
Solidor $^{\circledR}$, Brasil) e exérese da lesão com auxílio de pinça Adson (Golgran ${ }^{\circledR}$, Brasil).

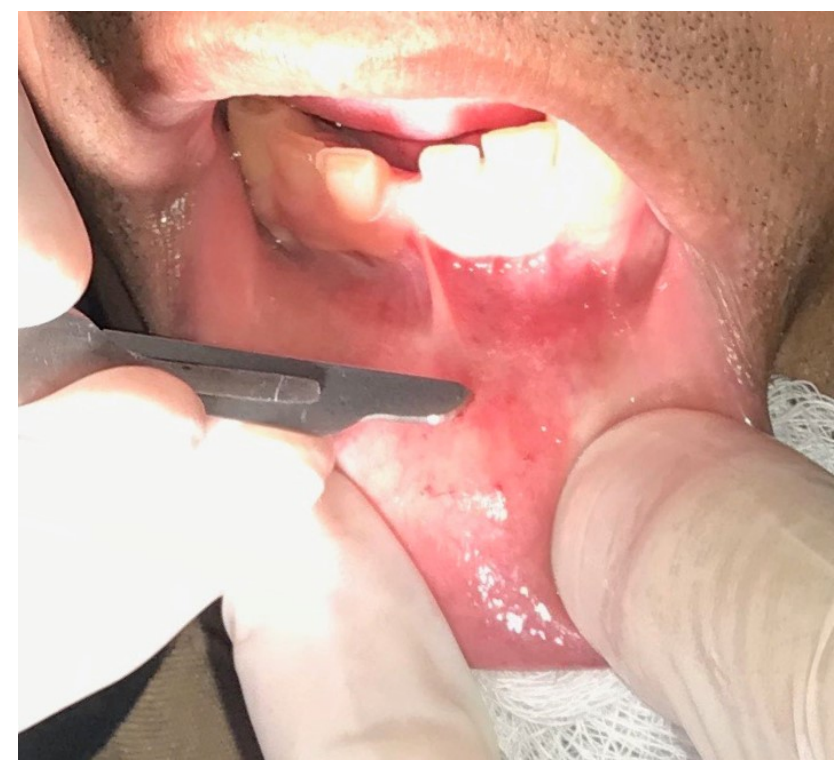

Figura 3: Incisão com lâmina de bisturi $\mathrm{N}^{\circ} 15$.

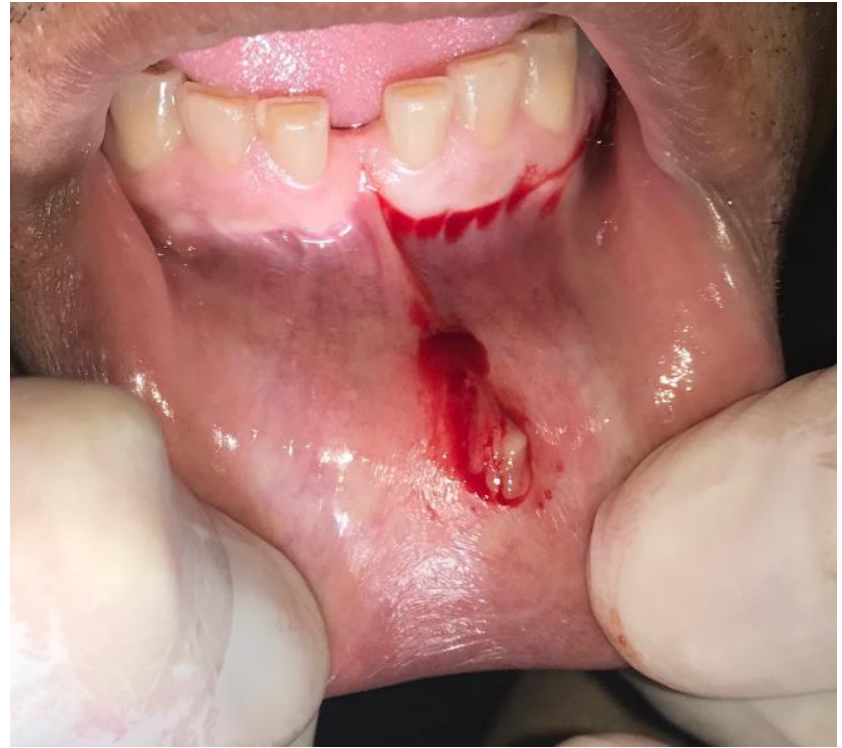

Figura 4: Mucocele exposta através da incisão cirúrgica.

Após retirada da mucocele, as glândulas salivares menores próximas à lesão foram removidas com auxílio de tesoura Íris de ponta reta $\left(\right.$ Golgran ${ }^{\circledR}$, Brasil) para evitar recidivas (Figuras 5 e 6 ).

Posteriormente, procedeu-se a divulsão dos tecidos com tesoura de Goldmam-Fox (Golgran ${ }^{\circledR}$, Brasil) de forma delicada para auxiliar na aproximação dos rebordos da ferida cirúrgica e melhorar a sutura.

Por fim, a síntese foi realizada por meio de sutura simples (Figura 7) com fio de seda 4.0 (Technew ${ }^{\circledR}$, Brasil). Foram dadas as orientações pósoperatórias. As suturas foram removidas sete dias após a intervenção cirúrgica.

Após três meses de acompanhamento, foi verificado que não houve recorrência da lesão e nem surgimento de novas lesões intra-orais.

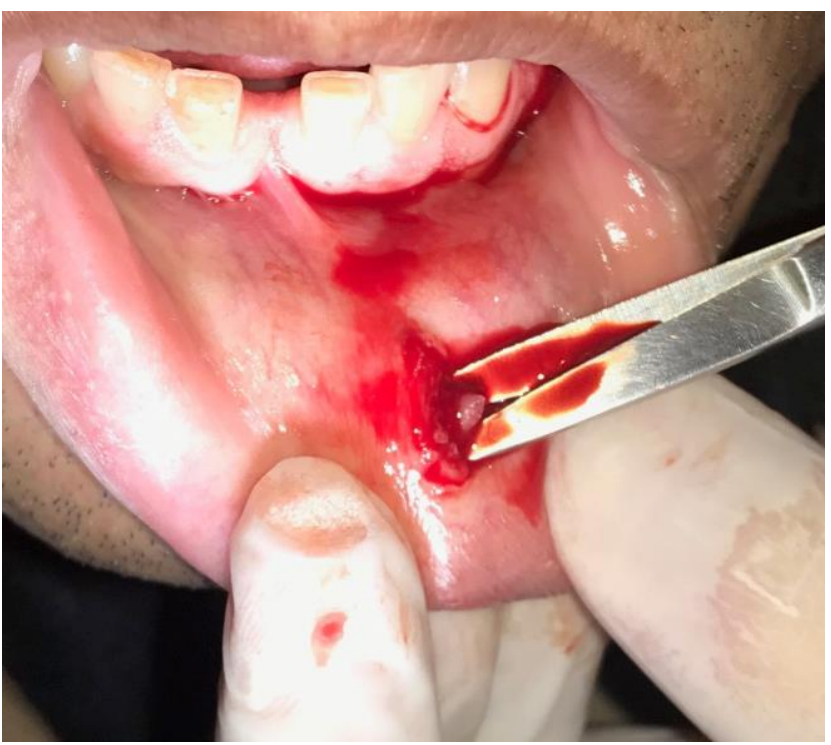

Figura 5: Remoção de glândula salivar menor associada.

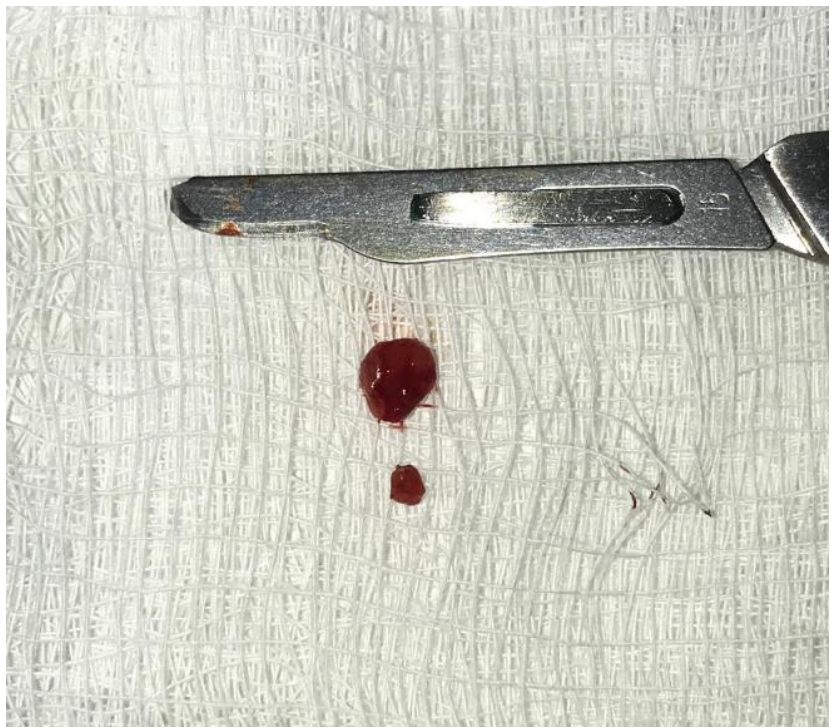

Figura 6 - Mucocele e glândula salivar menor removidas

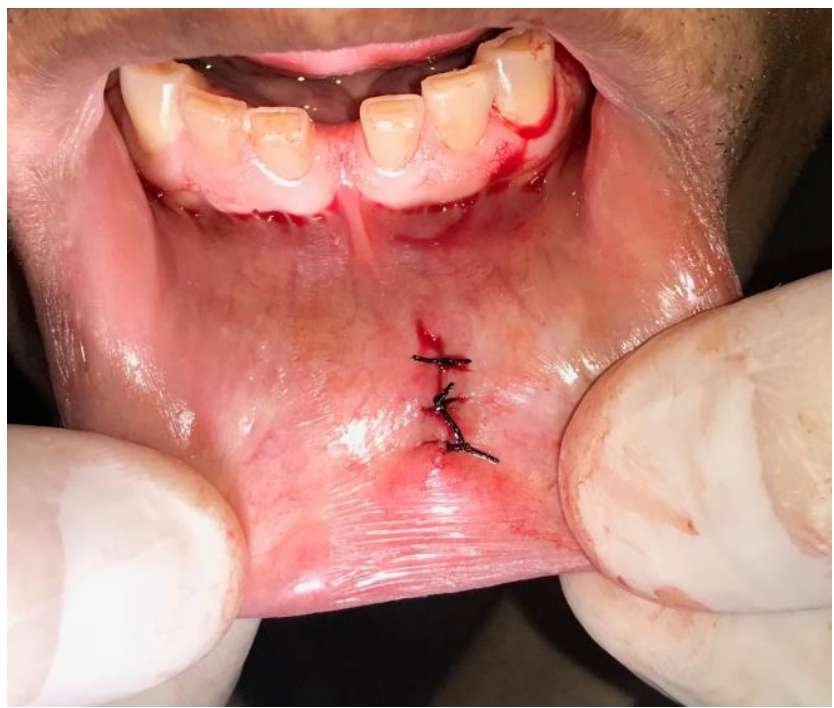

Figura 7 - Sutura em ponto simples

\section{DISCUSSÃO}

A maioria dos casos de mucocele oral é identificada pelos cirurgiões-dentistas durante os 
exames de rotina, ou mesmo pelos próprios pacientes por conta do aumento de volume causado pela retenção de saliva ${ }^{13}$. O seu diagnóstico é principalmente baseado na história e aparência clínica que inclui uma rápida aparição, localização específica, história de trauma, coloração semelhante à mucosa oral ou levemente azulada, consistência macia e flutuação ${ }^{14}$.

Cerca de $75 \%$ dos casos de mucocele possui menos de $1 \mathrm{~cm}$ de diâmetro, mas existem alguns casos descritos de lesões maiores, com 2-3 cm. De acordo com a literatura, quanto à incidência, não há diferença entre os sexos, embora sendo mais frequentes na segunda década de vida ${ }^{15,16}$.

Um estudo histopatológico é fundamental para confirmar o diagnóstico e também para garantir que $o$ tecido danificado foi completamente enucleado. Um dos diagnósticos diferenciais para mucocele são os fibromas, que também se caracterizam por lesão benigna de origem mesenquimal, entretanto a sua localização é, na maioria das vezes, a mucosa oral. Outra lesão que pode ser confundida com mucocele é o granuloma piogênico, cuja etiologia também pode ser um fator irritante $^{16}$. Histologicamente, a mucocele de extravasamento mostra-se como uma cavidade circunscrita por tecido conjuntivo e submucoso, com estiramento do epitélio. A parede dessa cavidade é forrada por tecido conjuntivo e não epitelial, caracterizando um pseudocisto ${ }^{5}$.

Embora a excisão cirúrgica da glândula de forma convencional ainda seja a principal opção terapêutica ${ }^{17}$, outras opções têm sido relatadas na literatura, tais como o uso de laser ${ }^{18,19}$, aplicação de corticosteroides no interior da lesão ${ }^{14,20}$, crioterapia $^{13}$, micromarsupialização ${ }^{8,21}$, entre outros.

A escolha por terapias menos invasivas deve-se ao fato de que algumas lesões de mucocele estarem localizadas em áreas onde uma incisão poderia, dependendo do diâmetro da lesão, deixar cicatrizes pós-operatórias como, por exemplo, no lábio. Para Fritz et al. ${ }^{22}$ o procedimento cirúrgico de exérese pode ser seguido por complicações como sangramento transoperatório, dificuldade na cicatrização da ferida cirúrgica, ruptura da síntese e a manutenção da esterilidade durante a cirurgia. No caso apresentado neste trabalho, não houve comprometimento estético após incisão tecidual, visto que a área acometida era a porção mais interna da mucosa labial inferior, assim como não houve outras intercorrências.

Alguns autores descreveram a eficácia do laser no tratamento de alterações dos tecidos orais através de terapia fotodinâmica ${ }^{23}$ e processos cirúrgicos $^{18,19}$. Chiżyński et al. ${ }^{24}$ reportaram um caso clínico onde realizaram a remoção de mucocele na porção ventral da língua em paciente adulto através do laser de dióxido de carbono $\left(\mathrm{CO}_{2}\right)$ com a potência de $3 \mathrm{~W}$ e descreveram os ótimos resultados quanto à elevada taxa de absorção de água e seus efeitos mínimos sobre os tecidos adjacentes.

Ganguly et al. ${ }^{25}$, Rankumar et al. ${ }^{19}$ e Bagher et al. $^{26}$ descreveram casos clínicos nos quais utilizaram o laser de diodo para remoção de mucocele em lábio inferior, onde elencaram uma variedade de efeitos benéficos de seu uso, como mínimo de anestesia, menos tempo processual, a boa visualização do campo cirúrgico, hemostasia, e o mínimo de carbonização em 45 dias de acompanhamento periódico. O laser de diodo (com comprimento de onda variando entre 800 e $980 \mathrm{~nm}$ ) é mal absorvido pelo tecido dental duro, é seguro, e bem indicado para cirurgias de tecido oral para corte, vaporização, curetagem, coagulação e hemostasia na região oral ${ }^{27}$.

A crioterapia também vem sendo utilizada para o tratamento da mucocele, onde ocorre o congelamento do tecido oral lesionado. É um procedimento bem tolerado que produz excelentes resultados e pode ser facilmente realizado no consultório $^{28}$. Aulakh et al. ${ }^{13}$ reportaram um caso clínico de mucocele na porção lateral esquerda do soalho bucal em que lançaram mão da crioterapia como modalidade de tratamento da lesão, obtendo bons resultados e apontando a ausência de desconforto pós-operatório, sítio cirúrgico sem sangue e excelentes resultados cosméticos como maiores benefícios.

A crioterapia destrói tecidos lesionados por aplicação do frio extremo através de diferentes agentes de produção criogênica, tais como o gás óxido nitroso ou spray de nitrogênio líquido. Temperaturas abaixo de $-20^{\circ} \mathrm{C}$ causam a morte celular $^{29}$.

Outro método para tratamento de lesões de mucocele é a utilização de corticosteroides injetáveis como uma alternativa à cirurgia. Mortazati et al. ${ }^{20}$ relataram um caso de mucocele em mucosa labial inferior onde associou as técnicas de micromarsupialização e injeção de fosfato de dexametasona no interior da lesão, obtendo resultados favoráveis. Baharvand et al. ${ }^{30}$ mostraram, em 7 casos de mucocele tratados com dexametasona, que 5 deles foram totalmente curados e 2 apresentaram redução em tamanho, sem nenhuma complicação pós-operatória. Sinha et al. ${ }^{14}$ documentaram o tratamento de 20 pacientes com mucocele utilizando a injeção intralesional de betametasona. Dos 20 casos, 18 deles apresentaram regressão completa da lesão, enquanto que os 2 casos restantes apresentaram diminuição no tamanho.

Os corticosteróides agem como potentes antiinflamatórios, inibindo a expressão de codificação de muitos genes inflamatórios (citocinas, quimiocinas, moléculas de adesão, enzimas e proteínas) e também podem aumentar a transcrição de genes que 
codificam proteínas anti-inflamatórias. ${ }^{31}$ Eles também agem como um agente esclerosante, causando $\mathrm{o}$ encolhimento dos dutos salivares dilatados ${ }^{30}$.

Delbem et al. ${ }^{21}$ descreveram o tratamento de mucocele em crianças utilizando a técnica de micromarsupialização, obtendo sucesso em $85 \%$ dos casos. O principal objetivo deste método é drenar o muco e reduzir o tamanho da lesão. Apresenta como vantagens a simplicidade na execução e o fato de ser relativamente indolor, com o mínimo de trauma. A técnica de micromarsupialização consiste em transpassar um fio de sutura pelo centro da lesão, o que permite o extravasamento do conteúdo mucoso até sua regressão ${ }^{32}$.

\section{CONCLUSÃO}

A mucocele é uma lesão comum e afeta a população em geral. Por essa razão, se torna importante conhecer as características clínicas das mucoceles e seu tratamento, para que se possa seguir um correto protocolo na prática clínica diária. Embora existam diferentes modalidades para o tratamento dessas lesões, a exérese da glândula salivar obstruída ainda é a técnica mais empregada, por ser um procedimento cirúrgico simples, seguro e que mostra bom prognóstico.

No caso clínico relatado nesse trabalho, após a remoção cirúrgica da lesão, os resultados se mostraram satisfatórios, com pós-operatório indolor, sem edema ou quaisquer outras queixas por parte do paciente. Não houveram recidivas ou aparecimento de novas lesões. A remoção completa da mucocele e glândulas salivares acessórias, bem como a ausência de recidivas, caracterizou o sucesso na abordagem do caso.

\section{REFERÊNCIAS}

1. Rao PK, Hegde D, Shetty SR, Chatra L, Shenai P. Oral mucocele: diagnosis and management. J Dent Med Med Sci. 2012; 2:26-30.

2. Laller S, Saini RS, Malik M, Jain R. An appraisal of oral mucous extravasation cyst case with mini review. J Adv Med Dent Sci Res. 2014; 2(2):166-70.

3. Chi AC, Lambert PR, Richardson MS, Neville BW. Oral mucoceles: a clinicopathologic review of 1,824 cases, including unusual variants. J Oral Maxillofac Surg. 2011; 69(4):1086-93.

4. Wu CW, Kao YH, Chen CM, Hsu HJ, Chen CM, Huang IY. Mucoceles of the oral cavity in pediatric patients. Kaohsiung J Med Sci. 2011; 27(7):276-79.

5. Regezi JA, Sciubba JJ. Cistos da boca. Patologia bucal: correlações clinicopatológicas. 6. ed. Rio de Janeiro: Guanabara Koogan; 2013.

6. Jani DR, Chawda J, Sundaragiri SK, Parmar G. Mucocele: a study of 36 cases. Indian J Dent Res.
2010; 21(3):337-40.

7. Sukhtankar LV, Mahajan B, Agarwal P. Treatment of lower lip mucocele with diode laser: a novel approach. Ann Dent Res. 2013; 2(Suppl 1):102-8.

8. Rodríguez H, DeHoyos Parra R, Cuestas G, Cambi J, Passali D. Congenital mucocele of the tongue: a case report and review of the literature. Turk J Pediatr. 2014; 56(2):199-202.

9. Re Cecconi D, Achilli A, Tarozzi M, Lodi G, Demarosi F, Sardella A et al. Mucoceles of the oral cavity: a large case series (1994-2008) and a literature review. Med Oral Patol Oral Cir Bucal. 2010; 15(4):e551-56.

10.Poulopoulos A, Andreadis D, Parcharidis E, Grivea I, Syrogiannopoulos G, Kolokotronis A. Salivary mucoceles in children and adolescents: a clinicopathological study. Global J Med Clin Case Rep. 2017; 4(1):11-4.

11.Ata-Ali J, Carrillo C, Bonet C, Balaguer J, Peñarrocha $M$, Peñarrocha $M$. Oral mucocele: review of the literature. J Clin Exp Dent. 2010; 2(1):e18-21.

12.More CB, Bhavsar K, Varma S, Tailor M. Oral mucocele: a clinical and histopathological study, J Oral Maxillofac Pathol. 2014; 18(5):72-6.

13.Aulakh KK, Brar RS, Azad A, Sharma S, Anand A, Jyoti B. Cryotherapy for treatment of mouth mucocele. Niger J Surg. 2016; 22(2):130-33.

14.Sinha R, Sarkar S, Khaitan T, Kabiraj A, Maji A. Nonsurgical management of oral mucocele by intralesional corticosteroid therapy. Hindawi Publishing Corporation. Int J Dent. 2016; Article ID 2896748:1-5. doi.10.1155/2016/2896748.

15. Kim JH, Park HY, Hong SP, Ahn SK. Concurrent occurrence of mucocele and pyogenic granuloma. Ann Dermatol. 2011; 23(Suppl 1):S108-10

16. Valério RA, De Queiroz AM, Romualdo PC, Brentegani LG, De Paula-Silva FW: Mucocele and fibroma: treatment and clinical features for differential diagnosis. Braz Dent J. 2013; 24(5):537-41.

17. Chaitanya P, Praveen D, Reddy M. Mucocele on lower lip: a case series. Indian Dermatol Online J. 2017; 8(3):205-7.

18. Vitale MC, Sfondrini MF, Croci GA, Paulli M, Carbone L, Gandini P et al. Diode laser-assisted surgical therapy for early treatment of oral mucocele in a newborn patient: case report and procedures checklist. Case Rep Dent. 2018, Article ID 3048429:1-6. doi.10.1155/2018/ 3048429.

19. Ramkumar S, Ramkumar L, Malathi N, Suganya R. Excision of Mucocele Using Diode Laser in Lower Lip. Case Rep Dent. Volume 2016; Article ID 1746316:1-4. doi.org/10.1155/2016/1746316

20. Mortazavi H, Baharvand M, Alirezaei S, NoorMohammadi R. Combination therapy in a large 
lower lip mucocele: a non-invasive recommended technique. Dent Hypotheses. 2014; 5(3):127-29.

21.Delbem, AC, Cunha RF, Vieira AE, Ribeiro LL. Treatment of mucus retention phenomena in children by the micro-marsupialization technique: case reports. Pediatr Dent. 2000; 22(2):155-58.

22.Fritz GR, Stern PJ, Dickey M. Complications following mucous cyst excision. J Hand Surg. 1997; 22(2):222-25.

23.Barcessat AR, Huang I, Rosin FP, Dos Santos Pinto Jr. D, Maria Zezell D, Corrêa L. Effect of topical 5-ALA mediated photodynamic therapy on proliferation index of keratinocytes in 4-NQOinduced potentially malignant oral lesions. $\mathrm{J}$ Photochem Photobiol B. 2013; 126:33-41. doi: 10.1016/j.jphotobiol.2013.06.011

24.Chiżyński A, Stelmach R, Osica P, Janas-Naze A. Mucocele in a 35-year-old patient: a case report. J Educ Health Sport. 2017; 7(12):274-79.

25.Ganguly R, Mukherjee M, Pal TK. Laser excision of a mucocele: a case report. J Int Clin Dent Res Organ. 2015; 7(2):168-70.

26.Bagher SM, Sulimany AM, Kaplan M, Loo CY. Treating mucocele in pediatric patients using a diode laser: three case reports. Dent J (Basel). 2018; 6(2). pii: E13. doi: 10.3390/dj6020013.

27. Amaral MB, Freitas IZ, Pretel H, Abreu MH, Mesquita RA. Low level laser effect after micromarsupialization technique in treating ranulas and mucoceles: a case series report. Lasers Med Sci. 2012; 27(6):1251-55.

28. Farah CS, Savage NW. Cryotherapy for treatment of oral lesions. Aust Dent J. 2006; 51(1):2-5.

29. Gaddam S, Sharma P. Advances in endoscopic diagnosis and treatment of Barrett's esophagus. J Dig Dis. 2010; 11(6):323-33.

30. Baharvand M, Sabounchi S, Mortazavi $H$. Treatment of labial mucocele by intralesional injection of dexamethasone: case series. J Dent Mater Tech. 2014; 3(3)128-33.

31.Barnes PJ. How corticosteroids control inflammation: quintiles Prize Lecture 2005. Br J Pharmacol. 2006; 148(3):245-54.

32. Shah SK, Le MC, Carpenter WM. Retrospective review of pediatric oral lesions from a dental school biopsy service. Pediatr Dent. 2009; 31(1):14-9.

\section{CONFLITO DE INTERESSES}

Os autores declaram não haver conflitos de interesse.

AUTOR PARA CORRESPONDENCIA

Bruno Firmino de Oliveira

bruno_firmino19@hotmail.com

Submetido em 08/06/2018

Aceito em 04/10/2018 